\title{
Resolving the circumstellar environment of the $B[e]$ star V921 Scorpii in the near-infrared with VLTI/AMBER ${ }^{\star}$
}

\author{
A. Kreplin ${ }^{1, \star \star}$, S. Kraus ${ }^{2}$, K.-H. Hofmann ${ }^{1}$, D. Schertl ${ }^{1}$, G. Weigelt ${ }^{1}$, and T. Driebe ${ }^{3}$ \\ 1 Max Planck Institut für Radioastronomie, Auf dem Hügel 69, 53121 Bonn, Germany \\ e-mail: akreplin@mpifr-bonn.mpg.de \\ 2 Department of Astronomy, University of Michigan, 500 Church St., Ann Arbor, MI 48109-1090, USA \\ 3 Deutsches Zentrum für Luft- und Raumfahrt, Königswinterer Strasse 522-524, 53227 Bonn, Germany
}

Received 21 March 2011 / Accepted 20 October 2011

\begin{abstract}
Aims. We study the AU-scale circumstellar environment of the unclassified B[e] star V921 Sco in the near-infrared. For interpreting the observations, we employ temperature-gradient disk models.

Methods. Using the near-infrared beam combiner instrument AMBER, we recorded spectrally dispersed (spectral resolution $R=35$ ) interferograms in the $H$ and $K$ bands. To obtain an improved calibration of the visibilities, we developed a method that is able to equalize the histograms of the optical path difference of target and calibrator. We fit temperature-gradient disk models to the visibilities and spectral energy distribution (SED) to analyze the circumstellar dust geometry.

Results. We derived a geometric ring-fit radius of $2.10 \pm 0.16$ mas in the $K$ band. If we adopt the distance of $1150 \pm 150 \mathrm{pc}$ reported elsewhere, we obtain a ring-fit radius of $2.4 \mathrm{AU}$, which is slightly smaller than the $3.5 \mathrm{AU}$ dust sublimation radius predicted by the size-luminosity relation. The fitted $H$-band radius of $1.61 \pm 0.23$ mas $(1.85 \mathrm{AU})$ is found to be more compact than the $K$-band radius. The best-fit temperature-gradient disk model has an inner disk radius of $\sim 1.45 \mathrm{AU}$, an inner-edge disk temperature $T_{0}=1533 \mathrm{~K}$, and a temperature-gradient exponent $q=0.46$ suggesting a flared disk geometry.

Conclusions. The distance and luminosity of V921 Sco are not well known. If we assume a distance of $1150 \pm 150 \mathrm{pc}$, we derive a ring-fit radius of $\sim 2.4 \mathrm{AU}$, which is approximately consistent with the computed temperature-gradient disk model with inner and outer ring radii of 1.45 and $8.5 \mathrm{AU}$, respectively. If the inner radius of V921 Sco is more compact than the sublimation radius, this compact observed size can be explained by emitting material (e.g., a gaseous disk) inside the dust sublimation radius, as suggested for several other $\mathrm{B}[\mathrm{e}]$ stars.
\end{abstract}

Key words. techniques: interferometric - stars: pre-main sequence - stars: individual: V921 Sco - circumstellar matter protoplanetary disks - accretion, accretion disks

\section{Introduction}

Circumstellar disks play a fundamental role in star and planet formation. Near-infrared spectro-interferometry with mas spatial resolution is able to resolve the inner AU-scale dust and gas disks of both young and evolved stars. The southern emissionline star V921 Sco (CD-42 11721 , MWC 865, Hen 3-1300) is an intermediate-mass object of spectral type B0 with $M_{\star}=$ 8-10 $M_{\odot}$ (Borges Fernandes et al. 2007). Surrounded by a small nebula (Glass \& Allen 1975), V921 Sco resembles objects belonging to the class of intermediate-mass young stellar objects, the Herbig Ae/Be stars (Herbig 1960). While Finkenzeller \& Mundt (1984) classified V921 Sco as a Herbig Be star, Hutsemekers \& van Drom (1990) point out its similarity to Be supergiants. Unfortunately, its stellar parameters and distances are not well known (de Winter \& The 1990: 400 pc; Shore et al. 1990: 2.6 kpc; Hillenbrand et al. 1992: $\sim 160$ pc; Habart et al. 2003: 800 pc; Borges Fernandes et al. 2007: 1.15 kpc).

^ Based on observations made with ESO telescopes at Paranal Observatory under program ID (MPG-VISA GTO): 079.C-0212(A).

$\star \star$ Member of the International Max Planck Research School (IMPRS) for Astronomy and Astrophysics at the Universities of Bonn and Cologne.
Recent studies have shown that V921 Sco is the most massive member of a small star cluster with about 33 member stars (Weidner et al. 2010). The embedded V921 Sco group is located $\sim 2$ pc away from an HII region (Wang \& Looney 2007). Submm observations by Mannings (1995) indicate a disk-dominated scenario based on the classification scheme by Hillenbrand et al. (1992). High-resolution optical spectra (Acke et al. 2005) have been interpreted as evidence of a flared disk geometry, leading to the classification of a Group I source (Meeus et al. 2001). Interferometric observations of the $\operatorname{Br} \gamma$ line indicate that the line-emitting region is only slightly smaller than the continuum-emitting region (Kraus et al. 2008a). These observations suggest that the $\mathrm{Br} \gamma$ line is emitted by a stellar wind, a disk wind, or a gaseous inner disk. The spectroscopic study by Borges Fernandes et al. (2007) favors an outflowing disk-wind scenario.

The structure of the paper is as follows. In Sect. 2, we describe the observations and data reduction. In Sect. 3, we discuss the temperature-gradient model used in our analysis. In Sect. 4, we briefly discuss our results and in Sect. 5 we present a conclusion. In the Appendix A, we describe a method that is able to equalize the histograms of the optical path differences of target and calibrator. In Appendix B we show $\chi^{2}$-maps of our temperature-gradient modeling. 
Table 1. Observation log of the VLTI/AMBER low-resolution $(R=35)$ observations of V921 Sco with the linear array A0-D0-H0.

\begin{tabular}{|c|c|c|c|c|c|c|c|}
\hline Data set & UT date & $\begin{array}{l}\text { UT time } \\
{[\mathrm{h}: \mathrm{m}]}\end{array}$ & $\begin{array}{l}\text { DIT } \\
{[\mathrm{ms}]}\end{array}$ & $\begin{array}{c}\text { Proj. baselines } \\
{[\mathrm{m}]}\end{array}$ & $\begin{array}{l}\text { PA } \\
{[\circ]}\end{array}$ & Calibrator & $\begin{array}{c}\text { UD size ( } K \text { band }) \\
{[\mathrm{mas}]}\end{array}$ \\
\hline A & $\begin{array}{l}2007-05-14 \\
2007-05-14 \\
\end{array}$ & $\begin{array}{l}06: 39 \\
06: 54 \\
\end{array}$ & $\begin{array}{c}100 \\
50\end{array}$ & $\begin{array}{l}31.5 / 63.0 / 94.5 \\
31.2 / 62.4 / 93.6\end{array}$ & $\begin{array}{l}75.9 \\
78.4 \\
\end{array}$ & $\begin{array}{l}\text { HD } 152040 \\
\text { HD } 152040\end{array}$ & $\begin{array}{l}0.69 \pm 0.24^{b} \\
0.69 \pm 0.24^{b}\end{array}$ \\
\hline B & $\begin{array}{l}2007-05-15 \\
2007-05-15\end{array}$ & $\begin{array}{l}04: 41 \\
04: 58\end{array}$ & $\begin{array}{c}100 \\
50\end{array}$ & $\begin{array}{l}31.6 \text { / 63.2 / } 94.8 \\
31.8 \text { / 63.6 / } 95.4\end{array}$ & $\begin{array}{l}56.4 \\
59.4\end{array}$ & $\begin{array}{l}\text { HD } 152040 \\
\text { HD } 145921\end{array}$ & $\begin{array}{l}0.69 \pm 0.24^{b} \\
0.96 \pm 0.01^{a}\end{array}$ \\
\hline
\end{tabular}

Notes. ${ }^{(a)}$ Taken from the CHARM 2 catalog (Richichi et al. 2005); ${ }^{(b)}$ Calculated with getCal available at http://nexsciweb.ipac . caltech. edu/gcWeb/gcWeb.jsp

\section{Observations, data reduction, and results}

V921 Sco was observed with the VLTI/AMBER instrument (Petrov et al. 2007) in two nights in May 2007 (see Table 1). The observations were carried out using three auxiliary $1.8 \mathrm{~m}$-telescopes (ATs) of the VLTI, arranged in the linear configuration A0-D0-H0. The AMBER instrument is a beam recombiner that records spectrally dispersed three-beam interferograms. Using AMBER in the low-spectral resolution (LR) mode $(R=35)$, we recorded $H$ - and $K$-band interferograms simultaneously. For data reduction, we used the AMBER data reduction package amdlib ${ }^{1}$ version 3.0 , which employs the P2VM algorithm (Tatulli et al. 2007).

We derived visibilities in ten spectral channels in the $K$ band (2.0-2.4 $\mu \mathrm{m}$ ) and also had sufficient flux in the $H$ band in data set B to extract visibilities between $1.65-1.75 \mu \mathrm{m}$ (see Fig. 1a). Unfortunately, no $H$-band visibilities could be derived from data set A because of the low signal-to-noise ratio (SNR).

Since the AMBER instrument also provides closure phases, possible asymmetries in the brightness distribution can be studied. In our observations, only the $K$-band data gave a reliable closure phase signal (Fig. 2). To calibrate the visibility and closure phase data, two calibration stars were observed (see Table 1). The wavelength calibration was derived from the broad telluric band gaps between the $J$ and $H$ bands and $H$ and $K$ bands. In order to improve the SNR of the derived calibrated visibilities, we selected $20 \%$ of the target and $20 \%$ of the calibrator interferograms with the highest fringe SNR (Tatulli et al. 2007). The $K$-band closure phase shown in Fig. 2 has values of approximately $-24.5 \pm 26.2^{\circ}$. Within the large error bars, this result is approximately consistent with a centro-symmetric brightness distribution.

Since the coherence length of AMBER LR observations is only approximately 60 to $80 \mu \mathrm{m}$, while the atmospheric piston in our data is on the order of $20 \mu \mathrm{m}$, we cannot neglect the influence of the optical path differences (OPDs) between the beams on the visibility calibration. To compensate for this effect, we developed a method which is able to partially correct systematic differences between OPD errors of the target and calibrator data. If the raw frames of the science target and the calibrator had nearly the same histogram of OPD values, the visibility transfer function of both would be nearly identical, and therefore would not much degrade the visibility calibration. Therefore, we improve our visibility calibration by performing an equalization of the OPD histograms of target and calibrator. A detailed description of the histogram equalization process is presented in Appendix A.

Since the position angle (PA) difference in the two data sets (Table 1 ) is only $\sim 19^{\circ}$ and the elongation of the two-dimensional

\footnotetext{
1 The AMBER - reduction package amdlib is available at: http://www.jmmc.fr/data_processing_amber.htm
}
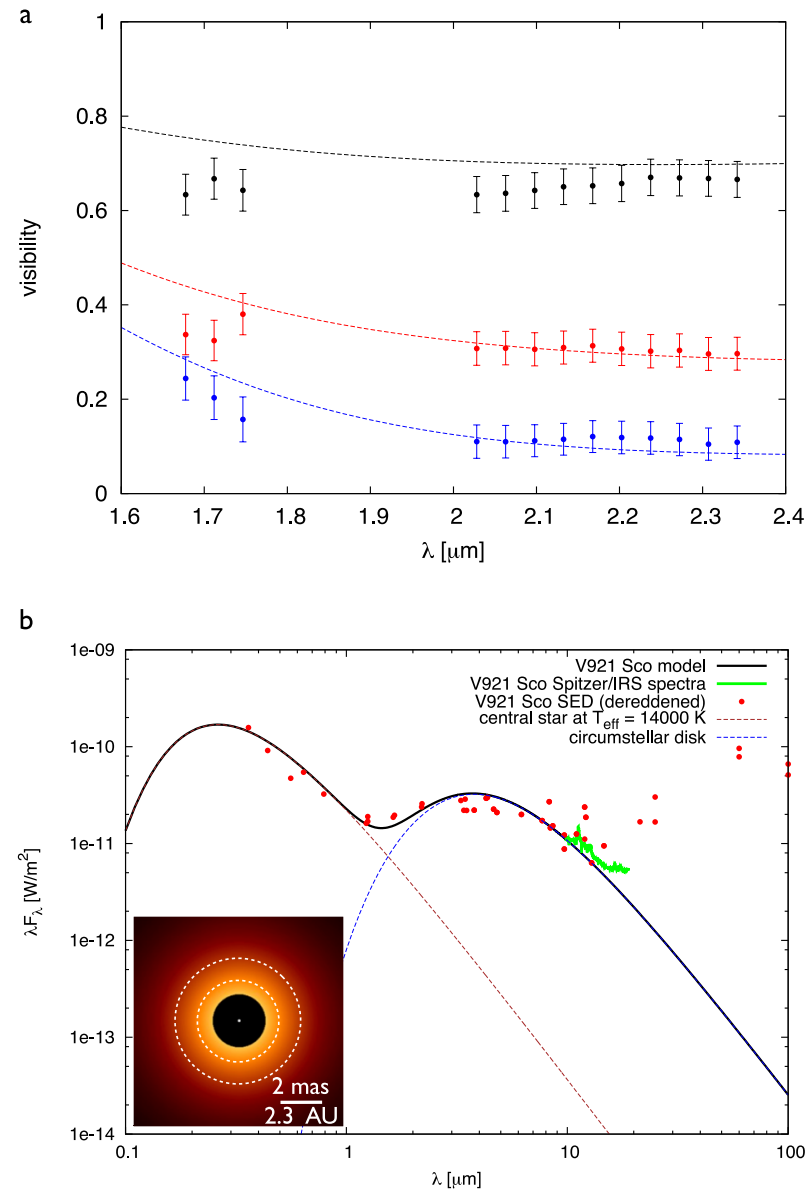

Fig. 1. AMBER visibilities, SED, and best-fit temperature-gradient model of V921 Sco. a) wavelength-dependent calibrated visibilities (baselines $31 \mathrm{~m}, 63 \mathrm{~m}, 95 \mathrm{~m}$ from top to bottom) and temperaturegradient model fits (dashed lines, see Sect. 3.2). b) available groundbased SED data (red points; Borges Fernandes et al. 2007), Spitzer spectrum (green line), and the model fit (black line). The inset shows the $K$-band intensity distribution of the best-fit temperature-gradient model (log scaling). The white dashed lines indicate the dust sublimation radii predicted by the size-luminosity relation for the temperatures of $1500 \mathrm{~K}$ (outer ring) and $2000 \mathrm{~K}$ (inner ring) using the stellar parameters reported by Borges Fernandes et al. (2007).

visibility is very small (see Fig. 3), we averaged the $K$-band visibilities of the data sets to enhance the SNR (see Fig. 1). $H$-band visibilities could only be extracted from data set B. In the upper part of Fig. 1, we show the obtained calibrated AMBER visibilities of V921 Sco in the $H$ and $K$ bands. The wavelengthaveraged $H$ - and $K$-bands visibilities are shown in Fig. 4. 
A. Kreplin et al.: Resolving the circumstellar environment of the B[e] star V921 Sco

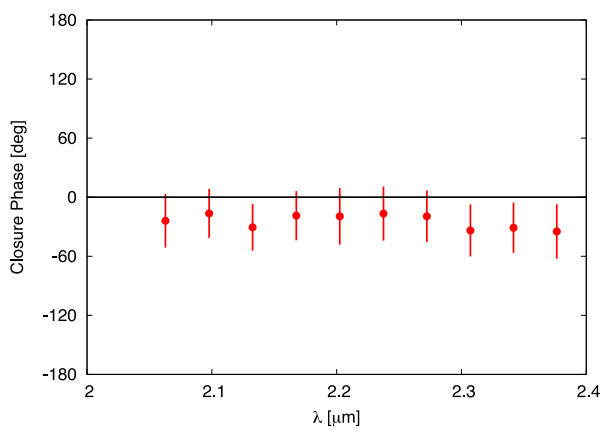

Fig. 2. Averaged $K$-band closure phases of the two data sets.

\section{Modeling}

\subsection{Geometric models}

To characterize the size of V921 Sco's circumstellar environment, we first fit three simple geometric models (uniform disk, Gaussian distribution, ring) to the observed visibilities (see Fig. 4). To take into account the central stellar emission, we modify the geometric visibility models according to

$V_{\text {tot }}=\frac{\left|F_{\star} V_{\star}+F_{\text {circ }} V_{\text {circ }}\right|}{F_{\star}+F_{\text {circ }}}$,

where $F_{\star}$ denotes the stellar flux, $F_{\text {circ }}$ the circumstellar flux component, and $V_{\text {circ }}$ and $V_{\star}$ denote the complex visibility of the circumstellar component and the star, respectively. Since the central star is unresolved by our measurements (the stellar radius is about 0.07 mas at $D=1150 \mathrm{pc}$ ), we set $V_{\star}=1$. To estimate the circumstellar-to-stellar flux ratio, we performed a spectral decomposition of the available SED data of V921 Sco. By fitting a Kurucz model (Kurucz 1979) to the stellar component of the dereddened SED, the excess flux was obtained as the difference of the observed flux to the Kurucz spectrum at $\lambda=2.2 \mu \mathrm{m}$ and $\lambda=1.7 \mu \mathrm{m}$ (we assumed $T_{\star}=14000 \mathrm{~K}$, $\log (g)=3.0$; Borges Fernandes et al. 2007). The uncertainty of the circumstellar-to-stellar flux ratio is approximately $10-20 \%$. We obtained a circumstellar-to-stellar flux ratio in the $K$ band of $f_{K}=F_{\text {circ }} / F_{\star}=10.8$ and in the $H$ band of $f_{H}=4.3$. The obtained geometric model fits are presented in Fig. 4. We derived a $K$-band ring-fit radius (i.e., inner radius of a ring with a width of $20 \%$ of the inner radius) of $2.10 \pm 0.16$ mas (corresponding to $2.41 \pm 0.18 \mathrm{AU}$ at a distance of $1150 \mathrm{pc})$. In the $H$ band $(\lambda=1.7 \mu \mathrm{m})$, we obtained a ring-fit radius of $1.61 \pm 0.23$ mas $(1.85 \pm 0.26 \mathrm{AU})$. For comparison we also calculated Gaussian HWHM and uniform-disk radii of V921 Sco's near-infrared emitting region: $r_{\mathrm{UD}, K}=4.23 \pm 0.45 \mathrm{AU}, r_{\mathrm{UD}, H}=3.74 \pm$ $0.33 \mathrm{AU}, r_{\text {Gauss }, K}=2.41 \pm 0.18 \mathrm{AU}, r_{\mathrm{Gauss}, H}=1.85 \pm 0.26 \mathrm{AU}$.

\subsection{Temperature-gradient model}

In a temperature-gradient disk model, the disk consists of many narrow rings emitting black body radiation (Hillenbrand et al. 1992; Eisner et al. 2007). The temperature of each ring at its radius $r$ is assumed to follow the power law:

$$
T(r)=T_{0}\left(\frac{r}{r_{\text {in }}}\right)^{-q}
$$

where $T_{0}$ is the temperature at the inner radius $r_{\text {in }}$ of the disk model. The power-law exponent $q$ is expected to be $q=3 / 4$

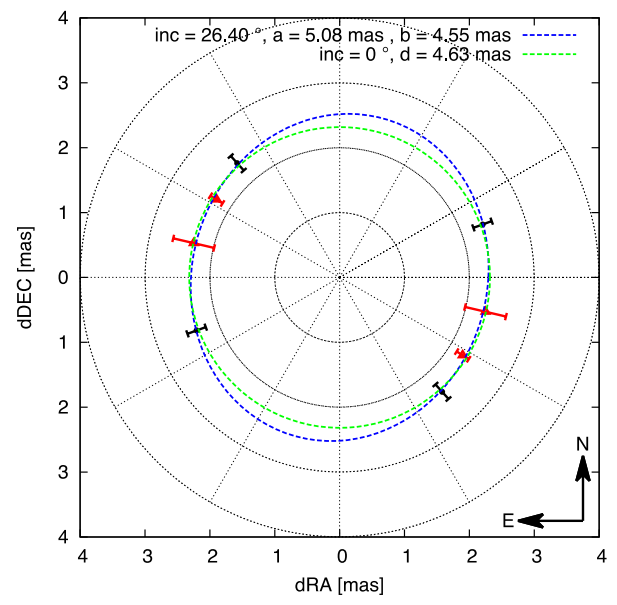

Fig. 3. Fit of an elliptical ring model (blue dashed line) to the AMBER visibilities (red triangles) and the visibilities reported by Kraus et al. (2008a) (black dots). The blue dashed line represents an ellipse fit with the major axis $a=5.08$ mas and minor axis $b=4.55$ mas. For comparison we also applied a circular ring fit with the diameter $d$ as a free parameter and obtained $d=4.63$ mas.

for a flat reprocessing disk and $q=0.5$ for a flared reprocessing disk (Kenyon \& Hartmann 1987). The temperature-gradient model disk is built up by several rings extending from an inner radius $r_{\text {in }}$ to an outer radius $r_{\text {out }}$. The small elongation shown in Fig. 3 suggests that V921 Sco's inclination angle (angle between the polar axis and the viewing direction) is small (in agreement with Borges Fernandes et al. 2007), since the major axis a and minor axis $b$ derived from the Kraus et al. (2008a) data combined with our new data are very similar ( $a \sim 5.0$ mas, $b \sim 4.6$ mas). Therefore, we decided to compute only face-on model images.

For the modeling and interpretation, we used the SED data and the stellar parameters of V921 Sco $\left(L_{\star}=10000 L_{\odot}, d=\right.$ $\left.1150 \mathrm{pc}, T_{\star}=14000 \mathrm{~K}, A_{V}=4.8, R_{\star}=17.3 R_{\odot}\right)$ reported in Borges Fernandes et al. (2007). The Spitzer data were taken from the archive ${ }^{2}$. The correction for interstellar extinction was performed following the extinction law by Cardelli et al. (1989).

The computed model visibilities and SEDs were compared to the observed visibilities and SED in the wavelength range up to $20 \mu \mathrm{m}$. A large number (more than $10^{8}$ ) of temperaturegradient models corresponding to all combinations of the four model parameters listed in Table 2 were calculated. In a first run, we scanned a broad parameter range (see Table 2 upper part) to find the global $\chi^{2}$-minimum. In a second modeling run, we focused on a narrower parameter range around the global $\chi^{2}$ minimum of our first run to increase the resolution around the best-fit model (see Table 2 lower part). We followed the strategy of fitting two-component models (i.e., unresolved star and a temperature power-law distribution) in order to keep the numbers of free parameters as low as possible. As best-fit model, we chose the model with the lowest $\chi_{\text {red }}^{2}$ value.

The best-fit model (Fig. 1; Table 2) has an inner disk radius of $\sim 1.5 \mathrm{AU}$ and an outer radius of $\sim 7.0 \mathrm{AU}$ with a temperature gradient of $q \sim 0.46$. The temperature at the inner disk radius $r_{\text {in }}$ was found to be $T_{0} \sim 1533 \mathrm{~K}$. This model is able to approximately reproduce both the observed visibility and SED data. The best-fit parameters of the temperature-gradient model

2 Observations were made with the Spitzer Space Telescope, which is operated by the Jet Propulsion Laboratory, California Institute of Technology under a contract with NASA. 

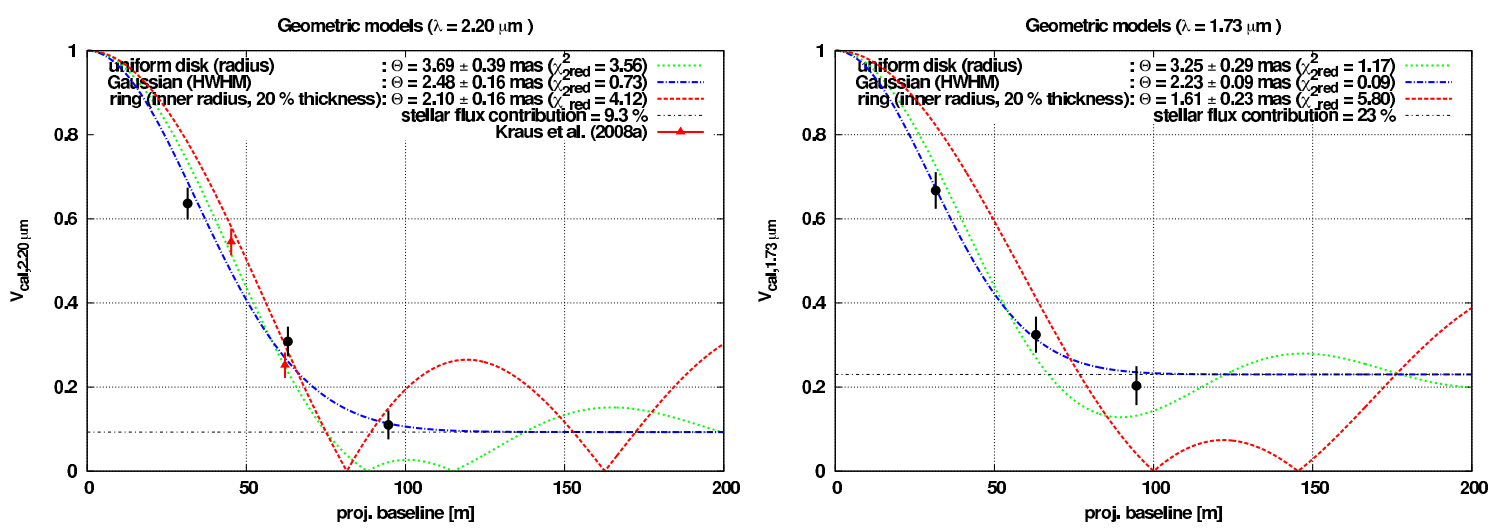

Fig. 4. Observed $K$ - (left) and $H$-band (right) visibilities at three different projected baselines and geometric model fits (UD, Gaussian, and ring models). In the left figure, we additionally plotted the $K$-band continuum visibilities reported by Kraus et al. (2008a) around the Br $\gamma$ line (red triangles).

Table 2. Scanned parameter space of the temperature-gradient modeling and best-fit model parameters.

\begin{tabular}{lcccc}
\hline \hline Scan range & $q$ & $r_{\text {in }}$ & $\Delta r=r_{\text {out }}-r_{\text {in }}$ & $T_{0}$ \\
& & {$[\mathrm{AU}]$} & {$[\mathrm{AU}]$} & {$[\mathrm{K}]$} \\
\hline wide & $0.05-3.05$ & $0.08-57.6$ & $0.08-57.6$ & $100-5100$ \\
narrow & $0.3-0.6$ & $0.9-3.2$ & $4.6-9.2$ & $1000-2000$ \\
\hline best fit & $0.46_{-0.04}^{+0.01}$ & $1.45_{-0.14}^{+0.06}$ & $7.05_{-0.91}^{+0.45}$ & $1533_{-20}^{+63}$ \\
\hline
\end{tabular}

Notes. First row: wide scan to find the global $\chi^{2}$-minimum. Each parameter was varied in 100 linear steps. Second row: narrow scan around the global minimum. Each parameter was varied in 30 linear steps. Bottom: best-fit parameters $\left(\chi_{\text {red }}^{2}=1.87\right)$ of the temperature-gradient model shown in Fig. 1.

are summarized in Table 2 (bottom). For visualization, we show $\chi^{2}$-maps in Appendix B.

\section{Discussion}

In order to compare the derived $K$-band ring-fit radius (inner radius of a model ring with a ring width of $20 \%$ of the inner radius) of $2.4 \pm 0.2 \mathrm{AU}$ (for the assumed distance of $1.15 \mathrm{kpc}$; Borges Fernandes et al. 2007) with other Herbig Ae/Be stars, we plotted V921 Sco into the size-luminosity relation (see Fig. 5) introduced by Monnier \& Millan-Gabet (2002) using the stellar luminosity of $L_{\star}=10000 L_{\odot}$ (Borges Fernandes et al. 2007). The size-luminosity diagram shows that the near-infrared ring-fit radii of most of the interferometrically observed objects roughly follow the predicted dust-sublimation radius.

If we assume that the Borges Fernandes et al. (2007) stellar parameters are correct, the position of V921 Sco in the sizeluminosity diagram suggests that the ring-fit radius is nearly equal to or only slightly smaller ( $\sim 1.5$ times) than the theoretical dust sublimation radius expected in the absence of radiationshielding material between the star and the disk (Monnier et al. 2005). If the inner radius of V921 Sco is really more compact than the sublimation radius, as suggested for several other $\mathrm{B}[\mathrm{e}]$ stars, this compact observed size can be explained by either (1) a bright additional disk component inside the dust sublimation region contributing to the total emission, or (2) an inner disk component that partially shields the radiation from the central star leading to a smaller dust sublimation radius (Akeson et al. 2005; Monnier et al. 2005; Kraus et al. 2008b; Benisty et al. 2010; Weigelt et al. 2011). The existence of such an inner disk is also supported by VLTI/AMBER observations of V921 Sco with medium spectral resolution that have shown that the $\mathrm{Br} \gamma$ line emitting gaseous region is more compact (approximately 1.4 times smaller) than the $K$-band continuum-emitting region (Kraus et al. 2008a). However, the distance and luminosity of V921 Sco remain disputed (see discussion in Borges Fernandes et al. 2007). Assuming a much closer distance, the radius could be even larger than the predicted dust-sublimation radius.

For our temperature-gradient modeling, we adopted the distance of $1.15 \mathrm{kpc}$ (Borges Fernandes et al. 2007) and the SED shown in Fig. 1. This temperature-gradient modeling has shown that a simple two-component model (star and one ring-shaped disk) is able to approximately reproduce both the visibilities and the SED. The best-fit inner disk radius of $r_{\text {in }}=1.45 \mathrm{AU}$ suggests an inner disk radius thats $\sim 2.4$ times more compact than the inner ring-fit radius predicted by the size-luminosity relation for a dust sublimation temperature of $1500 \mathrm{~K}$. This small inner radius of the disk can be explained by the disk structure (inner shielding disk component) discussed in the previous paragraph. The temperature-gradient exponent of $q=0.46$ suggests a flared disk geometry consistent with the classification of a group I source (Acke et al. 2005). However, since distance and luminosity are very uncertain (Borges Fernandes et al. 2007), it is not yet possible to decide whether the inner radius of the V921 Sco disk is really smaller than the dust sublimation radius.

\section{Summary and conclusion}

The unclassified B[e] star V921 Sco was observed with low spectral resolution in the $H$ - and $K$-bands using VLTI/AMBER. The following results were obtained.

- In order to improve the calibration of the target visibility, we developed a method that is able to partially correct systematic differences between the OPD errors of the target and the calibrator data. This method equalizes the histograms of the optical path differences of target and calibrator and can lead to smaller visibility calibration errors.

- The $H$ - and $K$-band-emitting regions have ring-fit radii of $\sim 1.85$ and $\sim 2.4 \mathrm{AU}$, respectively (at a distance of $d=$ $1150 \mathrm{pc}$ ). If the adopted distance and luminosity reported in Borges Fernandes et al. (2007) are correct, Fig. 5 shows that our derived $K$-band ring-fit radius of $2.4 \mathrm{AU}$ is nearly equal to or about $\sim 1.5$ times smaller than the 3.5 AU dust sublimation radius predicted by the size-luminosity relation for 


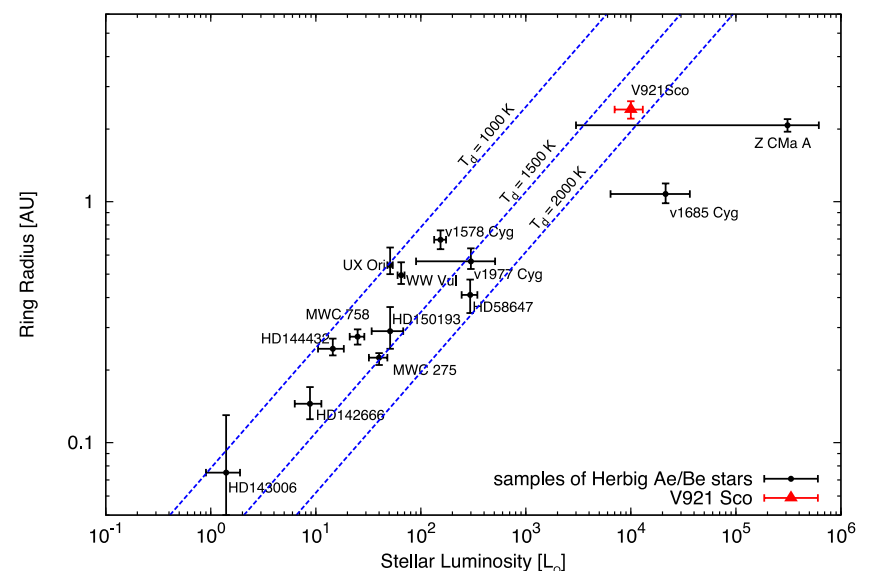

Fig. 5. Size-luminosity diagram of a sample of Herbig Ae/Be stars (adopted from Monnier et al. 2005). The $K$-band ring-fit radius of V921 Sco determined in this paper is plotted as a red triangle. The dotted blue lines represent model curves for three different dust sublimation temperatures. The plotted red error bar represents the parameter uncertainty reported in Borges Fernandes et al. (2007), but the total uncertainty is probably larger.

the dust temperature $T_{\mathrm{D}}=1500 \mathrm{~K}$ (if there is no radiationshielding material between the star and the disk).

- Within the error bars, the $K$-band closure phase is nearly consistent with zero, indicating an almost point-symmetric object, in agreement with the low inclination suggested in earlier studies (Borges Fernandes et al. 2007; Kraus et al. 2008a).

- The presented temperature-gradient modeling (star + disk) provides a circumstellar disk model that starts at an inner radius $r_{\text {in }}=1.45 \mathrm{AU}$ with a temperature of $T_{0}=1533 \mathrm{~K}$.

Assuming the stellar parameters reported by Borges Fernandes et al. (2007) are correct, both our geometric and the temperaturegradient modeling results suggest that the near-infrared flux of V921 Sco is emitted from a slightly more compact region than predicted by the size-luminosity relation. This small size might be explained by an inner disk made up of gas or refractory grains (Malbet et al. 2007; Tannirkulam et al. 2008; Kraus et al. 2008b; Benisty et al. 2010; Weigelt et al. 2011). However, since distance and luminosity are very uncertain (Borges Fernandes et al. 2007), it is not yet possible to decide whether the inner radius of the V921 Sco disk is really smaller than the dust sublimation radius. More detailed measurements with an improved position angle coverage are needed to better constrain the 2D brightness distribution of the circumstellar environment of V921 Sco. Furthermore, complementary observations in the mid-infrared would improve our knowledge of the disk parameters of V921 Sco considerably, since they would be able to probe a larger temperature gradient.

Acknowledgements. A. Kreplin was supported for this research through a stipend from the International Max Planck Research School (IMPRS) for Astronomy and Astrophysics at the Universities of Bonn and Cologne. This research has made use of NASA's Astrophysics Data System Bibliographic Services.

\section{Appendix A: Equalization of the histograms of target and calibrator to obtain improved calibrated visibilities}

The measured raw visibility of an interferogram depends on the instantaneous optical path difference (OPD), in particular, when a

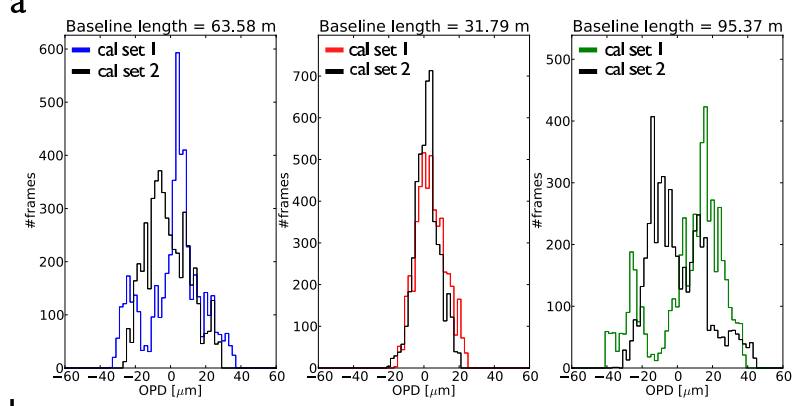

b
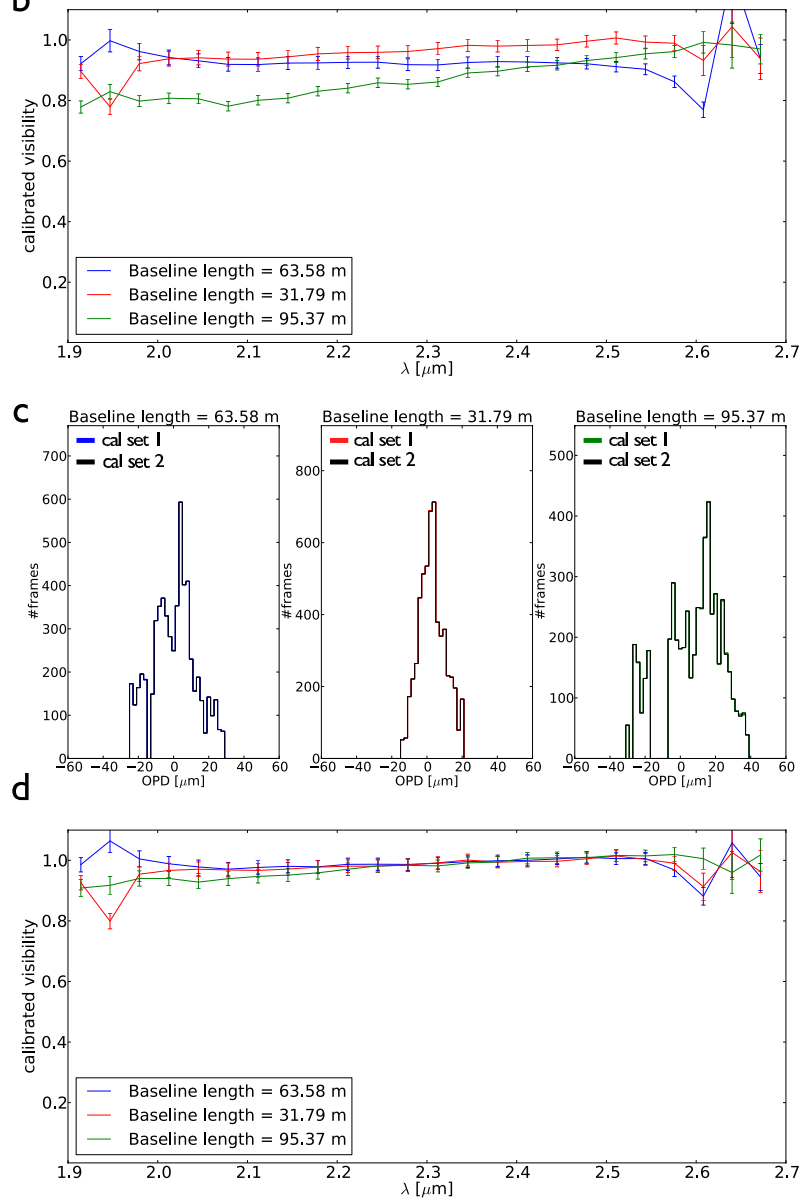

Fig. A.1. Test of the OPD histogram equalization with two different data sets of the calibrator HD 127753 . In this test, one of the two calibrator data sets plays the role of the "target data set". One expects that a visibility value of 1.0 is derived for this "test target", since the difference in the projected baselines of these two data sets is smaller than $1 \mathrm{~m}$. a) OPD histograms of the two data sets (big histogram differences of the interferograms obtained with the $95 \mathrm{~m}$ baseline). b) Computed visibilities obtained without OPD histogram equalization (i.e., histogram in panel a; the $95 \mathrm{~m}$ baseline shows the biggest deviation from the expected value 1.0). c) OPD histogram obtained after the histogram equalization was applied to the data sets (equalization is only applied to OPD bins that contain more than $20 \%$ of the peak number of interferograms to reject bins with low SNR). d) Computed visibilities obtained after the OPD histogram equalization was applied.

the coherence length is short, e.g., $70 \mu \mathrm{m}$ as for AMBER observations with a low spectral resolution of $R=35$. Therefore, the histograms of the target and calibrator OPD values should be kept as similar as possible during the observations. However, histogram differences often cannot be avoided because of the OPD drifts caused by errors of the OPD model or other reasons. 
If histogram differences cannot be avoided during data recording, one can try to equalize the OPD histograms of the target and calibrator by suitable software tools to minimize the visibility calibration error (as demonstrated in Fig. A.1). We want to note that the quality of the OPD histogram equalization of course depends on the obtained accuracy of the OPD estimation. Various effects can lead to corruptions of the OPD estimation, for example, noise in the interferograms of faint targets, telescope vibration (discussed below), signal degradation if an extended object is larger than the interferometric field-of-view, or any physical process that generates asymmetries in the interferometric signal.

The intensity distribution of a two-telescope AMBER interferogram can be described by (Tatulli et al. 2007):

$$
\begin{aligned}
i(\alpha, \lambda)= & \left\{I_{1}(\lambda)+I_{2}(\lambda)+2 \sqrt{I_{1}(\lambda) I_{2}(\lambda)}\right. \\
& \left.\times \operatorname{Re}\left[\Gamma_{J} V^{12}(\lambda) \mathrm{e}^{\mathrm{i}\left(\Phi_{12}(\lambda)+\phi_{p}^{12}(\lambda)\right)} \mathrm{e}^{\mathrm{i} 2 \pi \frac{B}{\lambda} \alpha}\right]\right\} E(\alpha, \lambda)
\end{aligned}
$$

where the position on the detector is described by the angular coordinate $\alpha, I_{1}(\lambda)$ and $I_{2}(\lambda)$ denote the photometric intensities, $V^{12}(\lambda)$ is the visibility of the object, $\Phi^{12}(\lambda)$ denotes the Fourier phase of the object, $\phi_{p}^{12}(\lambda)$ is a atmospheric phase difference between the two telescopes, given by $\phi_{p}^{12}(\lambda)=\frac{2 \pi}{\lambda} p$, where $p$ is the atmospheric piston, $B$ is the combination baseline in the exit pupil plane, $E(\alpha, \lambda)$ denotes the envelope function of the interferogram; i.e., the PSF of a single telescope. $\Gamma_{J}:=\exp \left(-\frac{1}{2} \sigma_{\phi_{\mathrm{h} f}^{p}}^{2}\right)$ represents the visibility reduction factor due to high-frequency piston variations (jitter), with the high-pass jitter denoted by $\phi_{\mathrm{h} f}^{p}$ (Colavita 1999). We note that the following idealized method cannot be used to correct for jitter effects, since their influence on the visibilities cannot be derived in general. Therefore, we assume that the jitter term has not changed between target and calibrator observations. To obtain the polychromatic interferogram, we integrate the monochromatic interferogram (Eq. (A.1)) from $\lambda_{0}-\frac{\Delta \lambda}{2}$ to $\lambda_{0}+\frac{\Delta \lambda}{2}$ over the bandwidth $\Delta \lambda$ around the center wavelength $\lambda_{0}$. If the band width $\Delta \lambda$ is small, we can assume that 1) the visibility and phase of the object are wavelengthindependent and that 2) the interferogram envelope $E(\alpha, \lambda)$, and the photometric intensities $I_{1}(\lambda)$ and $I_{2}(\lambda)$ are approximately $E\left(\alpha, \lambda_{0}\right), I_{1}\left(\lambda_{0}\right)$ and $I_{2}\left(\lambda_{0}\right)$, respectively. The integration over lambda leads to

$$
\begin{aligned}
& i\left(\alpha, \lambda_{0}\right) \approx\left\{I_{1}\left(\lambda_{0}\right)+I_{2}\left(\lambda_{0}\right)+2 \sqrt{I_{1}\left(\lambda_{0}\right) I_{2}\left(\lambda_{0}\right)} \operatorname{sinc}\left(\frac{p+B \alpha}{\lambda_{0} R}\right)\right. \\
& \left.\times \Gamma_{J} V^{12}\left(\lambda_{0}\right) \cos \left(\Phi_{12}\left(\lambda_{0}\right)+\frac{2 \pi}{\lambda_{0}}(p+B \alpha)\right)\right\} \Delta \lambda E\left(\alpha, \lambda_{0}\right)
\end{aligned}
$$

with the spectral resolution $R=\frac{\lambda_{0}}{\Delta \lambda}$ and coherence length $l_{\mathrm{c}}=$ $\lambda_{0} R$. According to the optical configuration of the AMBER instrument (Robbe-Dubois et al. 2007), the maximum baseline length of the AMBER exit pupil is $B_{\mathrm{M}}=3.9 D$, where $D$ is the diameter of a single pupil. Due to the envelope function $E\left(\alpha, \lambda_{0}\right)$, the maximum size of the interferogram is $\Delta \alpha=\frac{\lambda_{0}}{D}$, where $D=40 \mathrm{~mm}$ is the pupil diameter of AMBER in the $K$ band. Since $B \alpha \leq B \Delta \alpha$ and therefore much smaller than the coherence length $l_{\mathrm{c}}$ in AMBER's low-resolution mode, the term
$B \alpha$ in the sinc function of Eq. (A.2) can be neglected, leading to

$$
\begin{gathered}
i\left(\alpha, \lambda_{0}\right) \approx\left\{I_{1}\left(\lambda_{0}\right)+I_{2}\left(\lambda_{0}\right)+2 \sqrt{I_{1}\left(\lambda_{0}\right) I_{2}\left(\lambda_{0}\right)} \operatorname{sinc}\left(\frac{p}{\lambda_{0} R}\right)\right. \\
\left.\times \operatorname{Re}\left[\Gamma_{J} V^{12}\left(\lambda_{0}\right) \mathrm{e}^{\mathrm{i}\left(\Phi_{12}\left(\lambda_{0}\right)+\phi_{p}^{12}\left(\lambda_{0}\right)\right)} \mathrm{e}^{2 \pi \frac{B}{\lambda_{0}} \alpha}\right]\right\} \Delta \lambda E\left(\alpha, \lambda_{0}\right) .
\end{gathered}
$$

Equation (A.3) shows that the fringe contrast of the target and calibrator interferograms is reduced by the factor $\operatorname{sinc}\left(\frac{p}{\lambda_{0} R}\right)$. In the standard AMBER data reduction process, the calibrated visibilities are calculated by dividing the averaged raw visibility of the target $V_{\text {raw }}^{\text {sci }}\left(\lambda_{0}\right)$ by the transfer function $T\left(\lambda_{0}\right)=\frac{V_{\text {raw }}^{\text {cal }}\left(\lambda_{0}\right)}{V_{\text {theo }}^{\text {cal }}\left(\lambda_{0}\right)}$. For the calibrated visibility one therefore gets

$$
V^{\text {sci }}\left(\lambda_{0}\right)=V_{\text {theo }}^{\text {cal }}\left(\lambda_{0}\right) \frac{V_{\text {raw }}^{\text {sci }}\left(\lambda_{0}\right)}{V_{\text {raw }}^{\text {cal }}\left(\lambda_{0}\right)} \text {. }
$$

For example, the raw target visibility of frame $i$ is $V_{\text {raw, } i}^{\text {sci }}=$ $\operatorname{sinc}\left(\frac{p_{i}}{\lambda_{0} R}\right) V_{\text {true }}^{\text {sci }}\left(\lambda_{0}\right)$ with the true target visibility $V_{\text {true }}^{\text {sci }}\left(\lambda_{0}\right)$ and the instantaneous OPD $p_{i}$. The average raw visibility of the object can be described by

$$
\begin{aligned}
V_{\text {raw }}^{\text {sci }}\left(\lambda_{0}\right) & =\frac{1}{N} \sum_{i=1}^{N} V_{\text {raw }, \mathrm{i}}^{\text {sci }}\left(\lambda_{0}\right) \\
& =\frac{1}{N} \sum_{i=1}^{N} \operatorname{sinc}\left(\frac{p_{i}}{\lambda_{0} R}\right) \Gamma_{J} V_{\text {true }}^{\text {sci }}\left(\lambda_{0}\right) \\
& =\sum_{k=-\frac{m}{2}}^{+\frac{m}{2}} \operatorname{sinc}\left(\frac{k \Delta p}{\lambda_{0} R}\right) \Gamma_{J} P^{\text {sci }}(k \Delta p) V_{\text {true }}^{\text {sci }}\left(\lambda_{0}\right) \\
& =\left\langle\operatorname{sinc}\left(\frac{p}{\lambda_{0} R}\right)\right\rangle_{\text {sci }} \Gamma_{J} V_{\text {true }}^{\text {sci }}\left(\lambda_{0}\right)
\end{aligned}
$$

The first two lines in Eq. (A.5) are the usual average over the measured data ( $N$ frames), whereas the last two lines give a description of this average with the expectation value notation, where $P^{\text {sci }}(k \Delta p)$ denotes the probability density function, which is identical to the normalized histogram of the OPD values in the data set of the target frames. The $m$ OPD bins (with bin width $\Delta p)$ are centered on the OPD value $k \Delta p$.

By defining $V_{\text {raw }}^{\text {cal }}\left(\lambda_{0}\right)$ in the same way as in Eq. (A.5), and neglecting other influences on the measured visibilities we can rewrite the calibrated visibilities by

$$
V^{\text {sci }}\left(\lambda_{0}\right)=V_{\text {theo }}^{\text {cal }}\left(\lambda_{0}\right) \frac{\left\langle\operatorname{sinc}\left(\frac{p}{\lambda_{0} R}\right)\right\rangle_{\text {sci }} \Gamma_{J} V_{\text {true }}^{\text {sci }}\left(\lambda_{0}\right)}{\left\langle\operatorname{sinc}\left(\frac{p}{\lambda_{0} R}\right)\right\rangle_{\text {cal }} \Gamma_{J} V_{\text {true }}^{\text {cal }}\left(\lambda_{0}\right)} .
$$

The calibration degradation is caused by the two sinc terms $\left\langle\operatorname{sinc}\left(\frac{p}{\lambda_{0} R}\right)\right\rangle_{\text {sci }}$ and $\left\langle\operatorname{sinc}\left(\frac{p}{\lambda_{0} R}\right)\right\rangle_{\text {cal }}$, which are in general not equal because of the different OPD histograms of the target and calibrator data (see Fig. A.1a). This can be avoided, if we are able to make the OPD histograms of target and calibrator approximately equal. This is done by weighting the frames: the OPD histograms of the target and calibrator data can be equalized, if for the OPD bin $\mathrm{k}$ the target frames are multiplied by the factor $P^{\mathrm{cal}}(k \Delta p) / P^{\mathrm{sci}}(k \Delta p)$ and the calibrator frames are multiplied with the factor 1 , or vice versa. As can be seen in the example of Fig. A.1a, the OPD histograms of science target and calibrator can be quite different, which can, for example, cause visibility errors on the order of $5 \%$ in unfavorable cases (see Fig A.1b). 
On the other hand, if equalized histograms are employed, the deviation of the derived visibilities from the expected value 1.0 is now slightly smaller, in particular, for the $95 \mathrm{~m}$ baseline, which has the biggest histogram difference (see Fig. A.1d). This and similar tests suggest that the obtained improvement depends on the histogram differences and on the SNR of the raw data. The goal of the presented histogram equalization method is to make the OPD histograms of the science target and the calibrator identical to obtain a better visibility calibration, as described below in more detail. The weighting of the frames, necessary for the OPD histogram equalization, is performed in the following way.

First we define the total number of frames in our data set for the science target $\left(n_{\text {tot }}^{\text {sci }}\right)$ and the calibrator $\left(n_{\text {tot }}^{\mathrm{cal}}\right)$ separately:

$n_{\mathrm{tot}}^{\mathrm{sci}}=\sum_{k} n^{\mathrm{sci}}(k \Delta p), \quad n_{\mathrm{tot}}^{\mathrm{cal}}=\sum_{k} n^{\mathrm{cal}}(k \Delta p), \Delta p=2 \mu \mathrm{m}$.

The expressions $n^{\text {sci }}(k \Delta p)$ and $n^{\text {cal }}(k \Delta p)$ denote the measured histograms, i.e. the number of target frames and calibrator frames in the $k$ th bin within the OPD range from $k \Delta p$ to $(k+$ 1) $\Delta p$, respectively.

We define the ratio of the total number of frames as

$\varrho=\frac{n_{\text {tot }}^{\text {sci }}}{n_{\text {tot }}^{\text {cal }}}$

After the equalization process, the new OPD histograms of the target and the calibrator, i.e. $n_{\mathrm{eq}}^{\mathrm{sci}}(k \Delta p)$ and $n_{\mathrm{eq}}^{\mathrm{cal}}(k \Delta p)$ have to fulfill

$\frac{n_{\mathrm{eq}}^{\mathrm{sci}}(k \Delta p)}{n_{\mathrm{eq}}^{\mathrm{cal}}(k \Delta p)}=\varrho$

for every bin $k$. There are two possible cases: 1) If either $n^{\text {sci }}(k \Delta p)=0$ or $n^{\text {cal }}(k \Delta p)=0$, then the frames in this OPD range will not be used for further reduction. 2) One of the following ratios in bin $k$ is true:

$$
\frac{n^{\mathrm{sci}}(k \Delta p)}{n^{\mathrm{cal}}(k \Delta p)}>\varrho \quad \text { or } \quad \frac{n^{\mathrm{sci}}(k \Delta p)}{n^{\mathrm{cal}}(k \Delta p)}<\varrho \quad \text { or } \quad \frac{n^{\mathrm{sci}}(k \Delta p)}{n^{\mathrm{cal}}(k \Delta p)}=\varrho .
$$

In the two first cases of Eq. (A.9) one can approximately equalize the histograms by duplicating a few of the frames in the calibrator bin or in the science target bin, respectively. The duplication frames were statistically chosen out of the corresponding bin. If the last case of Eq. (A.9) is already true, this specific bin will not be altered. The number of duplicated frames $\Delta n_{k}$ is calculated for the first case of Eq. (A.9) by

$$
\begin{gathered}
\frac{n_{\mathrm{eq}}^{\mathrm{sci}}(k \Delta p)}{n_{\mathrm{eq}}^{\mathrm{cal}}(k \Delta p)}=\frac{n^{\mathrm{sci}}(k \Delta p)}{n^{\mathrm{cal}}(k \Delta p)+\Delta n_{k}}=\varrho \\
\longrightarrow \Delta n_{k}=\frac{n^{\mathrm{sci}}(k \Delta p)-n^{\mathrm{cal}}(k \Delta p) \varrho}{\varrho}
\end{gathered}
$$

and for the second case of Eq. (A.9) by:

$$
\begin{gathered}
\frac{n_{\mathrm{eq}}^{\mathrm{sci}}(k \Delta p)}{n_{\mathrm{eq}}^{\mathrm{cal}}(k \Delta p)}=\frac{n^{\mathrm{sci}}(k \Delta p)+\Delta n_{k}}{n^{\mathrm{cal}}(k \Delta p)}=\varrho \\
\longrightarrow \Delta n_{k}=\varrho n^{\mathrm{cal}}(k \Delta p)-n^{\mathrm{sci}}(k \Delta p) .
\end{gathered}
$$

To match the frame ratio, the equalization algorithm will duplicate a few frames (not delete frames). Therefore the weighting factor described above will always be greater than 1. Correcting the ratios in each bin, we finally end up with roughly the same OPD probability density function for both the science target and the calibrator, which leads to the same fringe contrast loss, i.e. $\left\langle\operatorname{sinc}\left(\frac{p}{\lambda_{0} R}\right)\right\rangle_{\text {sci }} \approx\left\langle\operatorname{sinc}\left(\frac{p}{\lambda_{0} R}\right)\right\rangle_{\text {cal }}$, in the raw visibilities. The described method will reduce the OPD influence on the final calibrated visibility $V^{\text {sci }}(\lambda)$ (see Fig. A.1). The histogram equalization was easy to perform with our data in spite of the noise in the data. We also tried other methods, for example, to correct the visibility of each individual interferogram by calculating the visibility loss caused by the individual OPD value of this interferogram. This method also worked but we found that the histogram equalization is easier to perform.

\section{Appendix B: $\chi^{2}$-maps}

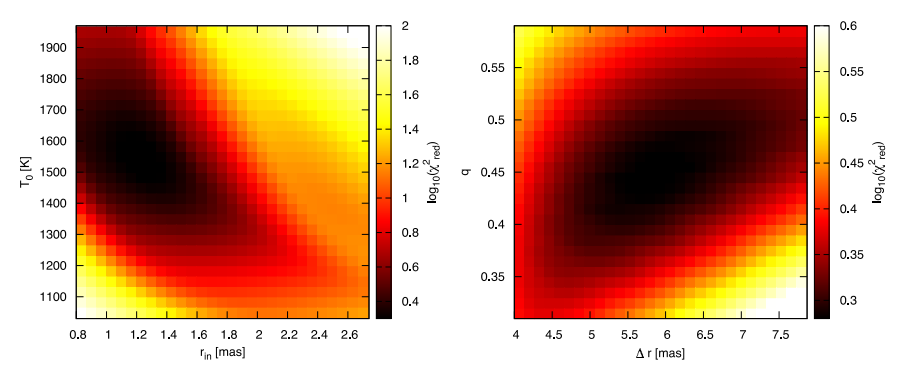

Fig. B.1. Reduced $\chi^{2}$-maps of the two-component temperature-gradient model. The $\chi_{\text {red }}^{2}$-value of each parameter combination in the maps represents the lowest $\chi_{\text {red }}^{2}$-value of all other parameter combinations.

\section{References}

Acke, B., van den Ancker, M. E., \& Dullemond, C. P. 2005, A\&A, 436, 209 Akeson, R. L., Walker, C. H., Wood, K., et al. 2005, ApJ, 622, 440 Benisty, M., Natta, A., Isella, A., et al. 2010, A\&A, 511, A74

Borges Fernandes, M., Kraus, M., Lorenz Martins, S., \& de Araújo, F. X. 2007, MNRAS, 377, 1343

Cardelli, J. A., Clayton, G. C., \& Mathis, J. S. 1989, ApJ, 345, 245

Colavita, M. M. 1999, PASP, 111, 111

de Winter, D., \& The, P. S. 1990, Ap\&SS, 166, 99

Eisner, J. A., Chiang, E. I., Lane, B. F., \& Akeson, R. L. 2007, ApJ, 657, 347 Finkenzeller, U., \& Mundt, R. 1984, A\&AS, 55, 109

Glass, I. S., \& Allen, D. A. 1975, The Observatory, 95, 27

Habart, E., Testi, L., Natta, A., \& Vanzi, L. 2003, A\&A, 400, 575

Herbig, G. H. 1960, ApJS, 4, 337

Hillenbrand, L. A., Strom, S. E., Vrba, F. J., \& Keene, J. 1992, ApJ, 397, 613

Hutsemekers, D., \& van Drom, E. 1990, A\&A, 238, 134

Kenyon, S. J., \& Hartmann, L. 1987, ApJ, 323, 714

Kraus, S., Hofmann, K., Benisty, M., et al. 2008a, A\&A, 489, 1157

Kraus, S., Preibisch, T., \& Ohnaka, K. 2008b, ApJ, 676, 490

Kurucz, R. L. 1979, ApJS, 40, 1

Malbet, F., Benisty, M., de Wit, W., et al. 2007, A\&A, 464, 43

Mannings, V. 1995, Ap\&SS, 224, 185

Meeus, G., Waters, L. B. F. M., Bouwman, J., et al. 2001, A\&A, 365, 476

Monnier, J. D., \& Millan-Gabet, R. 2002, ApJ, 579, 694

Monnier, J. D., Millan-Gabet, R., Billmeier, R., et al. 2005, ApJ, 624, 832

Petrov, R. G., Malbet, F., Weigelt, G., et al. 2007, A\&A, 464, 1

Richichi, A., Percheron, I., \& Khristoforova, M. 2005, A\&A, 431, 773

Robbe-Dubois, S., Lagarde, S., Petrov, R. G., et al. 2007, A\&A, 464, 13

Shore, S. N., Brown, D. N., Bopp, B. W., et al. 1990, ApJS, 73, 461

Tannirkulam, A., Monnier, J. D., Harries, T. J., et al. 2008, ApJ, 689, 513

Tatulli, E., Millour, F., Chelli, A., et al. 2007, A\&A, 464, 29

Wang, S., \& Looney, L. W. 2007, ApJ, 659, 1360

Weidner, C., Kroupa, P., \& Bonnell, I. A. D. 2010, MNRAS, 401, 275

Weigelt, G., Grinin, V. P., Groh, J. H., et al. 2011, A\&A, 527, A103 